\section{Misinterpretations of imagery-induced McCollough effects: A reply to Finke}

\author{
JACK BROERSE \\ Mount Gravatt College of Advanced Education \\ Mount Gravatt 4122, Australia
}

and

BORIS CRASSINI

University of Queensland, St. Lucia 4067, Australia

In commenting on our paper (Broerse \& Crassini, 1980), Finke (1981) examines the relevance of our data to "the proposal that imagined and observed patterns are functionally equivalent." His examination consists of questioning the rationale and reinterpreting the data of our first experiment. He then discusses some methodological differences between our second experiment and those of Finke and Schmidt $(1977,1978)$. On the basis of this examination, Finke concludes that "the results of the study by Broerse and Crassini do not provide serious evidence against the functional equivalence of imagined and observed patterns." We wish to reply to Finke's comments by reiterating some aspects of our original report that are pertinent to the points he raises. Moreover, since Finke seems to have missed the thrust of the conclusions in our original report, we welcome the opportunity to restate our interpretation of the data in relation to the issue of functional equivalence between imagined and observed patterns.

In our first experiment, we investigated the influence of self-reported imagery ability on the magnitude of colored aftereffects (CAEs) produced with physically present induction stimuli. The objection raised by Finke to this study seems to be his failure to find any relationship between self-reported imagery ability and perceptual performance on tasks very different from CAE production-namely, a peripheral visual acuity task (Finke \& Kosslyn, 1980) and a prism adaptation study (Finke, 1979). Implicit in this objection is the assumption that neural mechanisms underlying perception of dots in peripheral retina and the neural mechanisms involved in prism adaptation share identical properties with the neural mechanisms involved in CAE production.

Direct reprint requests to Jack Broerse, Department of Ps. chology and Special Education, Mount Gravatt College of Advanced Education, Messines Ridge Road, Mount Gravatt 4122, Australia, or to Boris Crassini, Department of Psychology, University of Queensland, St. Lucia 4067, Australia.
This is an empirical question answered in part by the results of our first experiment. We interpreted these results as suggesting that there may be two types of CAEs: one produced by physically present induction stimuli (and insensitive to observers' self-reported imagery ability) and the other produced by imagined induction stimuli limagery-induced colored aftereffects, IICAEs; reported by Finke and Schmidt $(1977,1978)$ to be sensitive to observers' self-reported imagery ability]. The rationale for our second study was to test this interpretation.

In our second experiment, we failed to find any evidence of IICAEs. We deliberately used a preposttest design to counteract the possibility that observers may have a bias toward a particular mode of color reporting (and not, as Finke states, as a control for observer's use of particular cognitive strategies; see below). We also used a non-forced-choice procedure (i.e., magnitude estimation) in which observers were able to report no color during CAE test phase. Finke suggests that these methodological differences (between our Experiment 2 and the studies of Finke \& Schmidt, 1977, 1978) may underlie our failure to generate IICAEs. We point out that we had already mentioned these methodological differences in our original report (see p. 566) and are at present in the process of determining whether such differences do in fact explain our failure. However, if, as Finke suggests, our failure to generate IICAEs is indeed due to the response procedures used, then this is itself evidence of further differences between CAEs and IICAEs and places in question any conclusions based on assumptions of close similarity between CAEs and IICAEs. We return to this issue in our conclusion.

In our second experiment, we also found no evidence to substantiate Finke's claim that "there is a strong tendency in this type of experiment for subjects to think that they are supposed to give as responses the same colors, in association with pattern orientation, that had been presented (or imagined) during adaptation procedures" (our italics). We found very few color responses of either the negative type (i.e., typical CAEs) or the positive type (i.e., Finke's association responses) following imagery induction, despite the constant reminder to observers during imagery adaptation of which orientation was associated with which color. While our non-forcedchoice procedures may have been insensitive in detecting IICAEs because of their weak nature (as suggested by Finke), we fail to see how our procedures would be insensitive to responses based on association strategies (given Finke's claim of a "strong tendency" to respond in this manner).

A further methodological point concerns our use 
of the Betts scale of self-reported imagery ability. Finke questions the usefulness of this scale and notes that "the importance of proper assessment and interpretation of individual differences in imagery vividness ... when conducting experiments on the relationship between imagery and perception." We support this caveat, as we did in our original report (pp. 566-567). In addition, we look forward to some reconciliation between statements made by Finke and his associates that there are clear and positive relationships between self-reported imagery ability and performance on imagery tasks and statements made by others who have reviewed the field. These reviewers claim that there are poor and/or inconsistent relationships between "pencil and paper" type indices of individual differences in imagery ability and objective performance on imagery tasks (see Ernest, 1977; White, Sheehan, \& Ashton, 1977).

A final methodological point concerns the elimination of subjects (up to 50\%) who did not respond appropriately in Finke and Schmidt's (1977, 1978) imagery induction conditions and who reported using association-type strategies during postexperimental questioning. We stated in our original report that this "seems an unnecessarily ad hoc method of culling data" (p. 566). We did not claim, as Finke implies, that postexperimental questioning about observers' use of strategies is "unnecessarily ad hoc," but that the "culling" of observers' data is. A less questionable procedure for investigating the role of strategies in the production of CAEs is to manipulate an observer's use of strategies within an experimental context. ${ }^{1}$

As a final comment, we wish to point out that we did not interpret the results of our experiments as constituting evidence either "for" or "against" (as implied by Finke) functional equivalence between imagined and observed patterns. Rather, we took our results to demonstrate that some basic assumptions about the comparability of CAEs and IICAEs had not been established: "It seems that IICAEs (1) are dependent on whether subjects imagine bars on physically present color patches or color on physically present bars, (2) are susceptible to different associative/cognitive strategies, and (3) are much weaker than CAEs produced normally" (p. 566). A further difference indicated by our data is that IICAEs cannot be produced reliably or, as Finke suggests, IICAEs may be dependent on the response procedures used to measure them. In either case, it is clear that there are a number of essential differences between CAEs and IICAEs. ${ }^{2}$ The major point of our original paper was that assumptions about the comparability of CAEs and IICAEs are fundamental in establishing the extent to which data about CAEs and IICAEs can constitute valid tests of functional equivalence between perception and imagery. Our own data, and in- deed those of Finke and Schmidt $(1977,1978)$, provide serious evidence against such assumptions of comparability. This issue must be resolved before the question of functional equivalence between imagined and observed patterns can be addressed.

\section{REFERENCE NOTE}

1. Broerse, J., \& Crassini, B. Can McCollough effects be modified by cognitive factors? Paper presented at the annual meeting of the Australian Psychological Association, Hobart, Australia, August 1979.

\section{REFERENCES}

Broerse, J., \& Crassini, B. The influence of imagery ability on color aftereffects produced by physically present and imagined induction stimuli. Perception \& Psychophysics, 1980, 28, 560-568.

ERNEST, E. I. Imagery ability and cognition: A critical review. Journal of Mental Imagery, 1977, 2, 181-216.

Finke, R. A. The functional equivalence of mental images and errors of movement. Cognitive Psychology, 1979, 11, 235-264.

Finke, R. A. Interpretation of imagery-induced McCollough effects. Perception \& Psychophysics, 1981, 30, 94-95.

Finke, R. A., \& Kossirn, S. M. Mental imagery acuity in the peripheral visual field. Journal of Experimental Psychology: Human Perception and Performance, 1980, 6, 126-139.

Finke, R. A., \& Schмidт, M. J. Orientation-specific color aftereffects following imagination. Journal of Experimental Psychology: Human Perception and Performance, 1977, 3, 599-606.

Finke, R. A., \& Schmidt, M. J. The quantitative measure of pattern representation in images using orientation-specific color aftereffects. Perception \& Psychophysics, 1978, 23, 515-520.

KunEN, S., \& MAY, J. G. Spatial frequency content of visual imagery. Perception \& Psychophysics, 1980, $28,555-559$.

Milewski, A. E., IAccino, J., \& SMITH, D. Checkerboardspecific color aftereffects: A failure to find effects of perceptual organization. Perception \& Psychophysics, 1980, 28, 329-336.

White, K., Sheehan, P. W., \& Ashton, R. Imagery assessment: A survey of self-report measures. Journal of Mental Imagery, $1977,1,145-170$.

\section{NOTES}

1. In a test situation in which achromatic test patterns do not appear colored, subjects in our procedure were permitted to respond "no color." In a forced-choice procedure (Finke \& Schmidt, 1977,1978 ), subjects were required to choose a color, despite the fact that no color may have been apparent. Finke and Schmidt then requested that their subject provide a written rationale outlining any strategies used in responding. Under these circumstances, it is not difficult to suggest a number of plausible accounts of subjects' introspective reports in terms of attempts to "double think" the experimenter and to provide an explicit strategy congruent (or indeed incongruent, in the case of hostile subjects) with what are thought to be the experimenter's expectations. Subjects' reports may be attempts to rationalize what to them appear to be inappropriate responses (i.e., reporting color when there is none). While such rationalization may not be the sole reason underlying the introspective reports, it is at least as plausible an interpretation as that adopted by Finke and his colleague. The elimination of some subjects on the assumption that one account is more plausible than others seems inappropriate. A better approach to investigate the possible role of cognitive strategies in the development of CAEs is to give subjects explicit directions to use different strategies while concurrently inducing (or not inducing) CAEs. We have found, for example, that subjects' color and no-color responses are directly dependent on the nature 
of the induction stimuli, irrespective of explicit instructions to subjects to use cognitive strategies that required them to construe in different ways the relationship between coior and orientation components of induction stimuli (Broerse \& Crassini, Note 1).

2. Further evidence of the differences between CAEs and IICAEs is provided by the data of Kunen and May (1980). In their first experiment, subjects underwent an adaptation procedure in which they were required to imagine a diamond checkerboard on, for example, a physically present homogeneous red patch in alternation with imagining a regular checkerboard on a green patch. Achromatic gratings used as test stimuli were reported red when vertical and green when oblique; that is, checkerboard IICAEs were contingent on edge orientation of the imagined induced checks (high spatial frequency components of the imagined checkerboards). This finding is unlike the case in which checkerboards and colors are physically present (Kunen \& May, 1980; Experiment 2). With such "real" adaptation, CAEs are found to be contingent on the fundamental spatial frequency components of the adaptation checkerboards (e.g., in the examples described above for imagined adaptation, achromatic test gratings would be reported green when vertical and red when oblique). That is, checkerboard CAEs are opposite to checkerboard IICAEs. This difference between checkerboard CAEs and IICAEs was eliminated when Kunen and May's subjects (Experiment 3) were sensitized to the fundamental frequency components of the adaptation checkerboards by blurring these during a preimagination phase. After this, subjects were asked to imagine blurred checkerboards on physically present color patches. The IICAEs generated following imagination of blurred checkerboards were like CAEs following adaptation to physically present, sharply focused stimuli.

In contrast with this pattern of results, Milewski, Iaccino, and Smith (1980) found that adaptation to physically present checker- board stimuli produced CAEs contingent on fundamental frequency information, irrespective of whether subjects perceptually organized the adaptation checkerboards into "vertical/horizontal" components (i.e., using high spatial frequency information) or "oblique" components (i.e, using fundamental spatial frequency information). Thus, it seems that the nature of color responses following adaptation to checkerboard stimuli depends on: (1) whether the stimuli are physically present (but not on how these are perceptually organized); (2) whether the stimuli are imagined clearly in focus (i.e., using high spatial frequency information); and (3) whether the stimuli are imagined as blurred (i.e., using fundamental spatial frequency information). Given these differing characteristics of CAEs and IICAEs induced with checkerboard stimuli, it seems presumptuous to us to conclude that such data tell us much about functional equivalence between perception and imagination.

Finally, there appears to be some inconsistency between Finke's (1981) proposition that Kunen and May's data may have been due to subjects' inability to hold in imagination all the spatial information available in a checkerboard and the claim by Milewski et al. (1980) that their subjects had little difficulty in perceptually organizing a checkerboard into either vertical/horizontal or oblique components-unless, of course, this represents a further difference among perception, perceptual organization, and imagination of checkerboard stimuli.

In summary, the data reported by Kunen and May lend further support to the conclusions expressed in the final paragraph of our reply.

(Manuscript received May 6, 1981; revision accepted for publication May 11, 1981.) 